\title{
Can Video Conferencing Be as Easy as Telephoning?-A Home Healthcare Case Study
}

\author{
Alan Taylor, Greg Morris, Jennifer Tieman, David Currow, Michael Kidd, Colin Carati \\ Faculty of Medicine, Nursing and Health Sciences, Flinders University, Adelaide, Australia \\ Email: alan.taylor@flinders.edu.au
}

Received 13 January 2016; accepted 5 March 2016; published 10 March 2016

Copyright (C) 2016 by authors and Scientific Research Publishing Inc.

This work is licensed under the Creative Commons Attribution International License (CC BY).

http://creativecommons.org/licenses/by/4.0/

(c) (i) Open Access

\begin{abstract}
In comparison with almost universal adoption of telephony and mobile technologies in modern day healthcare, video conferencing has yet to become a ubiquitous clinical tool. Currently telehealth services are faced with a bewildering range of video conferencing software and hardware choices. This paper provides a case study in the selection of video conferencing services by the Flinders University Telehealth in the Home trial (FTH Trial) to support healthcare in the home. Using pragmatic methods, video conferencing solutions available on the market were assessed for usability, reliability, cost, compatibility, interoperability, performance and privacy considerations. The process of elimination through which the eventual solution was chosen, the selection criteria used for each requirement and the corresponding results are described. The resulting product set, although functional, had restricted ability to directly connect with systems used by healthcare providers elsewhere in the system. This outcome illustrates the impact on one small telehealth provider of the broader struggles between competing video conferencing vendors. At stake is the ability to communicate between healthcare organizations and provide public access to healthcare. Comparison of the current state of the video conferencing market place with the evolution of the telephony system reveals that video conferencing still has a long way to go before it can be considered as easy to use as the telephone. Health organizations that are concerned to improve access and quality of care should seek to influence greater standardization and interoperability though cooperation with one another, the private sector, international organizations and by encouraging governments to play a more active role in this sphere.
\end{abstract}

\section{Keywords}

Telehealth, Home Care, Video Conferencing, Standards, Interoperability 


\section{Introduction}

\subsection{Delivering Healthcare to the Home}

The Flinders University Telehealth in the Home trial (FTH trial) was as an action-research initiative evaluating the use of video conferencing alongside other applications used by hospital based outreach services. The trial introduced telehealth services in the following clinical areas:

1) Community based palliative care. Patients and their carers received video-conferencing and remote monitoring services from a specialist palliative care nurse using a tablet device (iPad), a self-assessment application to record their symptoms regularly, and electronic devices and scales to monitor their physical activity and weight.

2) Home-based rehabilitation services for the elderly at home and in aged care facilities [1]. Patients were monitored by a therapist remotely, who made video calls as required, and also had access to rehabilitation and speech therapists using a tablet device (iPad), supported by a self-assessment application to record their health status and an exercise tracking device to monitor their physical activity.

Clinical care was delivered from the Repatriation General Hospital, Adelaide, South Australia to FTH trial participants using internet protocol (IP) based video conferencing. Connectivity between the hospital and participants was achieved through three mechanisms, the Australian National Broadband Network (NBN), mobile data services (Telstra 3G/4G), and the dedicated South Australian Health Digital Telehealth Network.

Both fixed line (NBN) connections and mobile data connections were used to provide access to participants. Mobile data services were able to immediately provide services to patients after discharges from acute settings, and were of sufficient quality to provide many of the services required in this trial. However, they were less reliable than fixed lines, and resulted in additional technical and clinical issues, and lower quality video conferencing.

The exploitation of consumer grade technologies to provide healthcare to people at home is potentially a game changer for telehealth services. Despite the ready availability of consumer products the implementation of telehealth services to the home using these technologies can be difficult. In particular the use of video conferencing into the home using everyday communications services and devices is not as simple as may be imagined. Consumers and clinicians are looking for applications that are easy to use while concerns about security and other factors drive the creation more complex and less usable solutions.

\subsection{Aim of This Case Study}

The paper discusses the impact on healthcare of poor interoperability between video conferencing solutions, provides a historical perspective on the development of interoperable telecommunications networks discusses of standards, or rather the lack of standards, which support the use of video conferencing in healthcare. This paper does not seek to make a direct comparison regarding the relative efficacy of telephone and video conferencing in healthcare service delivery. Rather, following an introduction in the following section to video conferencing technologies, this paper traces the experience of FTH trial in reconciling the needs of healthcare service delivery to the home and the consequent technological requirements, with the features of the video conferencing solutions currently available on the market.

An introduction to video conferencing technologies is followed by an outline of the methods and requirements used when selecting video conferencing technology to support home healthcare. The technology selection requirements (usability, cost, compatibility, interoperability, performance and privacy), the criteria used to evaluate each requirement and the process of product elimination that occurred are described. The discussion of the resulting outcome for the FTH trial highlights issues around video connectivity in healthcare, reliability, usability, quality and compares the development of video communications with that of voice communications (telephony) in the context of health informatics standardization.

\section{Video Conferencing Technologies for Home Healthcare}

\subsection{Software}

The core components of a video conferencing solution are video and audio processors (known as codecs) running on the computer hardware that encode and decode the video and audio, and a system to manage the identi- 
fication, addressing, connection or disconnection of calls to a number of users. Methods of identifying users and equipment include use of telephone numbers, equipment IP addresses, and user email addresses.

Software for video conferencing may be installed on devices as part of the operating system, plugin applications or as part of major application and collaboration software or a Web browser [2]. Most devices, including smart phones, tablets and personal computers currently available are now powerful enough to support video conferencing decoding and display software. The question is no longer the speed of the processor in the device, but whether the software used by a video conferencing application can be installed and will work on a device.

\subsection{Codecs}

The key issue for users is that different codecs are used by different software developers and may be mutually incompatible. For instance Skype uses one type of codec, while Facetime will use another, and the International Telecommunications Union (ITU) standards based systems use a third type called H.323 [3]. This last type of codec was one of the first codecs designed to operate over the internet using IP protocols, and has a large installed base of hardware based devices. The H.323 codec has been updated over the years and supplemented by a more advanced codec called H.264 [4]. Audio codecs are well established and many are freely available without licensing issues to developers. Video codecs are a different matter. Because video is information rich, codecs must compress video information into a small stream of information before transmission over a network. Since video compression is more demanding major companies have invested heavily in codec development, or licensing rights.

Sometimes cooperation between the commercial sponsors of each codec type becomes possible when all parties have more to gain by compromising or collaborating. For instance, the inclusion of video conferencing software in a web browser is the aim of an open license free collaboration supported by the developers of web browsers. This collaboration, called Web Real Time Communications (WebRTC) [5] offers web application developers the ability to write video conferencing applications that run within browsers, without requiring plugins, downloads or other installations [6]. An agreement [7] has been reported between Mozilla, Cisco and Google to allow browsers with WebRTC functionality to support competing codecs, in Firefox and Chrome browsers. It should be noted that WebRTC is an application programming interface that provides access to voice and video codecs already built into modern web browsers. Access to a server on the internet to identify and pass on signaling or addressing information to or from another browser is still required.

\subsection{Addressing}

Addressing is the Achilles heel of video conferencing. In the dim past video conferencing was possible on telephone lines, and users just required knowledge of the telephone number belonging to the distant party. The telephone system uses the dialed digits to find a connection to the distant party. The rise of the internet and the adoption of the internet to carry video conferencing have changed that model. Addressing of video conferencing calls now requires the association of an IP address on the internet with either a telephone number, or use of the Internet Engineering Task Force Session Initiated Protocol (SIP) [8] together with a Universal Address Identifier using a system known as ENUM [9].

In telephone systems each telephone exchange uses a computer based addressing and signaling system called "Signaling System 7" that permits international routing of telephone calls. A telephone exchange can be viewed as analogous to a server on the internet that processes voice or video communications. However in the case of video communications over the internet, international standards, regulations and agreements have to date failed to enable the same functionality for video communications. The ITU H.323 standards do in principle provide a solution for managing addressing over the internet using a gatekeeper device and this solution is widely used in internet IP telephony. However, telecommunications companies have not implemented this solution for video communications, possibly because they did not see a large enough market to justify additional investment in internet capacity for video traffic.

\subsection{Internet Video}

Since transmission of audio and video information over the internet is now possible, the software that sets up calls and establishes the flow of information between video conferencing users can now be located anywhere in 
the internet, on any server and does not have to be run by, or connect to a traditional telecommunications provider. This has enabled many services to be set up to provide video conferencing connections between users that are allowed to join a server on the internet. Fees are charged to users for registration with a server, and users are then able to make video conferencing calls to other users on the same service. These servers become the basis for video conferencing clouds on the internet.

\section{Methods}

\subsection{Available Video Conferencing Solutions}

During 2013 and 2014 the available video conferencing options on the market included:

- Hardware based conferencing based on the ITU H.323 standards [3] and IETF recommendations using the SIP recommendations running over IP. The hardware may be part of a telephone like product, or contained in a standalone box.

- Software based clients running on personal computing devices using proprietary protocols and user directories, the best known of which is Skype.

- Software based clients from major software vendors running on personal computing devices using proprietary protocols and user directories, the best known brands at the time being Microsoft Lync, Cisco Jabber, Vidyo, Apple Facetime, Polycom, and Lifesize, all of which use SIP based protocols to a greater or lesser degree.

- Software based clients running on personal computing devices using open source, SIP based communications protocols. These are available as software plugins or applications.

- Software based clients running in Web browsers, again using SIP protocols. Most of these clients require the installation of plugins except for WebRTC clients in Firefox and Chrome browsers where video conferencing is already built into the browser software.

\subsection{Requirements for Home Video Conferencing}

Rehabilitation and palliative care clinical teams in the FTH trial emphasized the need to provide video conferencing solutions appropriate to and affordable by people aged over 65 or those who are frail. Consequently the equipment and applications were selected to conform to the following requirements:

- usability and reliability — easy and reliable for clinicians and patients to use;

- cost and compatibility — built with compatible consumer grade applications and equipment;

- interoperability — with existing health sector ICT infrastructure;

- performance - can sustain adequate video and audio quality over a variety of networks; and

- privacy — can protect the privacy of patients.

Each video conferencing solution available on the market was assessed against a pragmatic set of criteria for each of the above requirements. In order to enable the reader to follow the process through which the eventual option was chosen, the selection criteria used for each requirement and the corresponding results are jointly described in the following section.

\section{Solution Selection Requirements, Criteria and Elimination Results}

\subsection{Usability}

Many elderly or frail patients and caregivers may not have the technical knowledge to navigate through complex interfaces and procedures [10]. A complex interface was considered to be an interface that required the patient to:

- configure or download software;

- enter login credentials each time an app was opened or device rebooted;

- enter a call or meeting ID to join conference; or

- navigate a busy interface, containing too many options/buttons (as found in most collaborative and social media based solutions).

Video conferencing solutions (Table 1) were assessed for usability on a 5-point rating scale, with 1 being bad and 5 being good. Each aspect of the application was given an initial score of 5, with a demerit point applied for 
Table 1. Jabber and Vidyo application usability.

\begin{tabular}{|c|c|c|}
\hline & Jabber & Vidyo \\
\hline Call initiation steps & Three & Three \\
\hline Simplicity & $\begin{array}{l}\text { Overall use of the application was simple. Red ' } X \text { ' } \\
\text { when an error occurred was confusing as appearance } \\
\text { made it seem like an interactive button. No option to } \\
\text { cancel sign-in process. This meant the application } \\
\text { appeared to be in a frozen state until the sign-in either } \\
\text { completed or timed out. }\end{array}$ & $\begin{array}{l}\text { Overall use of the application was simple. } \\
\text { User directory online status is confusing for } \\
\text { 'legacy' devices as they are displayed as an } \\
\text { online user “ }\end{array}$ \\
\hline Clarity of use & $\begin{array}{l}\text { Tool bar fades after } 5 \text { seconds and it is not obvious } \\
\text { how to display toolbar again. Connection/Online } \\
\text { status isn’t obvious. Can be confusing as to why calls } \\
\text { are not working. }\end{array}$ & $\begin{array}{l}\text { Tool bar fades after } 5 \text { seconds and it is not } \\
\text { obvious how to display toolbar again. }\end{array}$ \\
\hline Ease of navigation & $\begin{array}{l}\text { Settings and dial pad icons are not obvious/clearly } \\
\text { marked }\end{array}$ & Buttons are clearly marked \\
\hline Graphic user interface & $\begin{array}{l}\text { Text is too small for the visually impaired. Answer } \\
\text { button is clearly labelled, colored and positioned. }\end{array}$ & $\begin{array}{l}\text { Text is too small for the visually impaired. Answer } \\
\text { button is clearly labelled and positioned. }\end{array}$ \\
\hline Sounds & $\begin{array}{l}\text { Unique ringing sound allows user to identify } \\
\text { incoming call with application } \\
\text { If iPad volume is muted - audio is automatically } \\
\text { enabled when entering a call }\end{array}$ & $\begin{array}{l}\text { Unique ringing sound allows user to identify } \\
\text { incoming call with application. } \\
\text { If iPad volume is muted, audio is automatically } \\
\text { enabled when entering a call }\end{array}$ \\
\hline Summary & $\begin{array}{l}\text { Jabber provides an easy to use interface with an } \\
\text { automatic sign in function, eliminating the need for } \\
\text { a patient to sign in. }\end{array}$ & $\begin{array}{l}\text { Vidyo provides an easy to use interface with an } \\
\text { automatic sign in function, eliminating the need for } \\
\text { a patient to sign in. On a reboot of the iOS device, } \\
\text { the patient is prompted with a login button, user } \\
\text { credentials are pre-filled. }\end{array}$ \\
\hline
\end{tabular}

each issue found. The aspects scored were: number of steps to initiate call, simplicity of application, clarity of use, ease of navigation via the graphical user interface and graphic user interface sounds.

Virtually all video conferencing software products require the user to install a vendor specific application or plugin to support their particular protocols and cloud based services. Because the FTH trial sought to minimize the user involvement in configuration, tablet devices with pre-installed software were lent to patients for the duration of their treatment.

\subsection{Cost and Compatibility}

On grounds of cost, the need to minimize the number of devices installed in a patient's home, and the requirement for software applications (clinically orientated) to support patients, the use of dedicated video conferencing hardware-based video products was ruled out. At the time of product selection no simple-to-use video conference phone equivalent to a desk telephone for the public switched telephone network was available. It should be noted that most videophones on the market are intended for use within large organizations, appear complex to operate and require connection to a vendor's management platform. Videophones also typically had screen sizes that were too small for the intended clinical purpose.

Software based video conferencing solutions, based on tablet computing devices were considered the cheapest option at the time. However, only Apple products were sold preconfigured with a video conferencing solution (Facetime). Initially Facetime was used successfully by palliative care patients prior to implementation of the chosen solution. Additional research (Table 2) indicated that many Android variants and some Windows devices were incompatible with many third party video conferencing applications.

\subsection{Interoperability}

The FTH trial aimed to provide video conference solutions which were interoperable with technologies already used by South Australian Health (SA Health), and private health care providers, since care provision crosses all these sectors. There are two dimensions to interoperability between networks, trust and technical compatibility. For instance, due to security policies, SA Health permits outbound video calls to other networks, but does not 
allow inbound calls. The SA Health network is built on dedicated endpoints and multipoint control units using ITU H.323 standards. Therefore all potential solutions (Table 3) had to be assessed as being ITU H.323 interoperable, containing a gateway or proxy to translate between systems, or operating through a multipoint control unit (MCU).

Gateways that would translate between some common consumer services such as Apple Facetime, and the Skype product (now owned by Microsoft) can be expensive even when accessed on a subscription basis. Therefore Facetime and Skype services were not further considered.

SA Health used Cisco Jabber as their internal video conferencing desktop client. The preferred tablet device for SA Health was the Apple iPad 4th Generation. One of the clinical applications for use by palliative care patients at home was restricted to use on an iPad. For these reasons and the limited availability of some video conferencing clients on some Android tablet devices the initial preference was for the Cisco Jabber product running on an Apple iPad.

Alternative products including Vidyo, Polycom, Lifesize and others were considered. All these options required the use of a proprietary vendor solution installed in an internet based cloud, or custom software installed in a Flinders University data center. The possibility of using the emerging vendor neutral WebRTC solution, running in a browser was considered, but at that time, no WebRTC application was available for the iPad.

\subsection{Performance}

For a given video conferencing device or software the audio and video performance is highly dependent on the characteristics of the transmission network. The transmission of audio requires the network to deliver audio information or packets within narrow time periods, and minimize the loss of packets. Video transmission is less sensitive to time delays, but requires much higher network capacity, because the coding of video picture information creates more information to transmit than audio encoding.

Field tests, reported elsewhere [11] were conducted in different physical locations with both Cisco Jabber and Vidyo applications. Users felt that Vidyo was able to sustain a video and audio stream of higher quality compared to that of Jabber in poor 3G signal areas. It was found that the Jabber client was unable to reconnect to the registration server after the signal dropped out until the patient was guided through rebooting the iPad. The Vidyo product was able to interoperate with video conferencing standard protocols through a Flinders University gateway, traverse organizational firewalls and maintain user registration, thus avoiding the need to reboot the iPad.

Table 2. Platform compatibility matrix for solution plugins.

\begin{tabular}{ccccc}
\hline Platform & Jabber & Vidyo & FaceTime & WebRTC \\
\hline Windows & Yes & Yes & No & Yes using Chrome, Internet Explorer with plugin \\
iOS & Yes & Yes & Yes & No \\
Mac OS & Yes & Yes & Yes & Yes using Chrome \\
Android & No & Yes & No & Yes using Chrome \\
\hline
\end{tabular}

Table 3. Solution interoperability.

\begin{tabular}{|c|c|c|c|}
\hline Connectivity & Jabber & Vidyo & Facetime \\
\hline Point-to-Point & $\begin{array}{l}\text { Yes } \\
\text { Jabber-Jabber } \\
\text { Jabber-H323 } \\
\text { Jabber-SIP (So far no) }\end{array}$ & $\begin{array}{l}\text { Yes } \\
\text { Vidyo-Vidyo } \\
\text { Vidyo-H323 (Vidyo Gateway) } \\
\text { Vidyo-SIP (Vidyo Gateway) }\end{array}$ & $\begin{array}{l}\text { Yes } \\
\text { Facetime to Facetime }\end{array}$ \\
\hline Case Conference with SA Health & $\begin{array}{l}\text { Yes } \\
\text { Jabber Multi-Point } \\
\text { Jabber-MCU }\end{array}$ & $\begin{array}{l}\text { Yes } \\
\text { Vidyo Multi-Point } \\
\text { Vidyo to MCU (VidyoProxy) }\end{array}$ & No \\
\hline Connectivity out of SA Health Network & $\begin{array}{l}\text { Yes } \\
\text { (Registered to Health) }\end{array}$ & $\begin{array}{l}\text { Yes } \\
\text { (VidyoProxy) }\end{array}$ & Only Facetime to Facetime \\
\hline Connectivity into SA Health Network & $\begin{array}{l}\text { No/Yes } \\
\text { (Requires booking on MCU) }\end{array}$ & $\begin{array}{l}\text { Yes } \\
\text { (VidyoProxy) }\end{array}$ & Only Facetime to Facetime \\
\hline
\end{tabular}




\subsection{Privacy}

Evaluation of the Vidyo conferencing system uncovered a critical risk to patient confidentiality. The Vidyo system is structured around the concept of video room tenancies. Vidyo allows any user within a tenancy to call another member in the same tenancy. While the chances of an individual patient being able to navigate the system and dial a random contact number were minimal, this was considered to be a critical risk to patient privacy that needed to be addressed.

It was therefore decided to implement a multi-tenant Vidyo environment as on-premises, solution hosted by Flinders University. The on-premises Vidyo system was set up with each patient account in their own tenancy, effectively isolating them. The clinicians' tenancy was setup to allow visibility of the patient tenancies, providing them the ability to view a patient's online status and to dial-out to the patients. A Flinders University hosted solution also had the advantage that no information related to patients could possibly travel outside Australia, ensuring full compliance with Australian privacy legislation.

\section{Discussion}

\subsection{A Process of Elimination}

The assessment process described above led the FTH trial to choose an Apple iPad device and a proprietary Vidyo video conferencing solution hosted by Flinders University. This outcome illustrates how solution designers by a process of elimination become gradually dependent on a few technology providers. The preference of another party (SA Health) for one tablet device, together with usability considerations led to choice of the Apple iPad tablet. This meant that only video conferencing applications that would run as plugins on the iPad (Apple Store “apps”) could be considered, because the Apple operating system (IOS) and Apple browser (Safari) did not permit use of the almost vendor neutral WebRTC option, which in any case was and still is an immature product.

\subsection{Video Connectivity in Healthcare}

Providers of integrated health care in both the acute and community health settings can benefit from being able to set up, book, and schedule video calls with other providers and with patients in a range of settings and organization. At present the need for interconnection of different providers is most pronounced within the operating boundaries of local health services that wish to integrate the provision of care. The need for national video conferencing connectivity in Australia is at present likely to be driven by the provision of healthcare advice to callers from any location in the country. In Australia the listing of telehealth (video) health service delivery as a payable Medical Benefits Scheme Item has created an opportunity for consultant specialists, general practitioners and patients to consult using video conferencing to any patient in the country that is living outside defined metropolitan areas.

Implementation of national video conferencing connectivity is complex. A range of technology choices (Figure 1) have to be considered when connecting consumers with organizations that have historically only used dedicated video conferencing endpoints within an enterprise environment. A particular casualty of the complexity resulting from vendor competition is the ability of a healthcare provider or patient to locate a provider who has compatible equipment and is willing to provide consultations using video conferencing.

Even when a willing provider is located with compatible equipment it is rarely possible for a patient to call a doctor directly or for a consultant specialist or general practitioner to place a video call to a colleague in another organization without manual intervention. Manual intervention often takes the form of users exchanging identities (such as in the case of Skype users), or emailing a web hyperlink which the distant user can click on to active a call. Video conferencing addresses can also be accessed from a directory such as the Australian College of Rural and Remote telehealth provider directory Medicine [12] and then that address must be entered in an address field before the video conferencing software can call a provider. In some cases a two stage process may be occur when a call is routed via a gateway to another organization before reaching the destination party. This situation is analogous to the manual telephone switchboard era where all phones calls had to be placed through an operator.

There are many reasons for this state of affairs. Firstly, video conferencing cloud businesses do not readily share directory information about their subscribers. Secondly, health organizations do not often share contact 


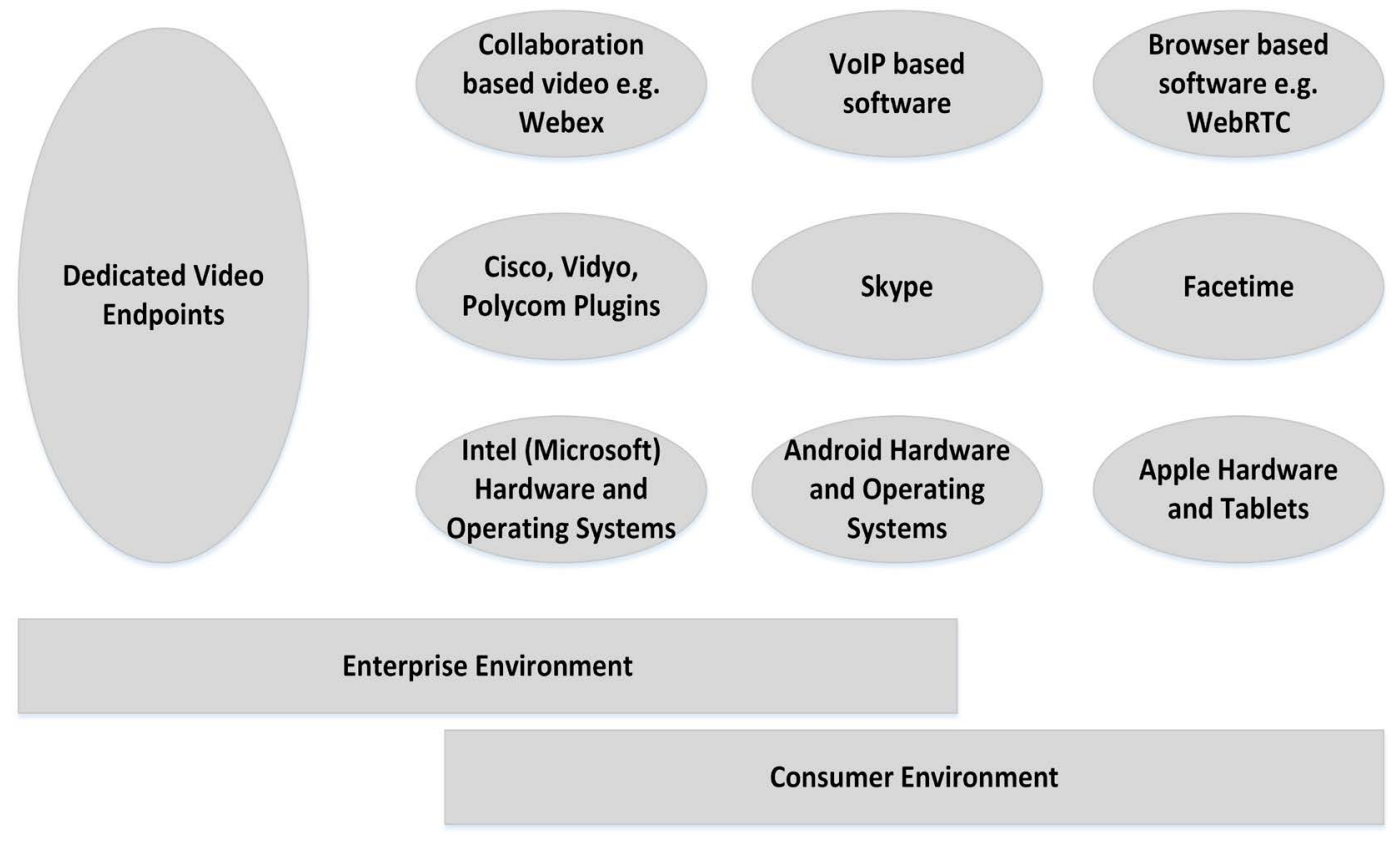

Figure 1. Video conferencing technology choices.

information for their health professionals, and prefer to channel patient contact through a telephone switchboard or a reception. Thirdly, video conferencing technology is still immature so it is rare to find a reception or switchboard that can transfer an incoming video call to the destination health professional. Fourthly, many cloud services operate on the basis of invitation to participate in a video conferencing session via an SMS or email, instead directly "calling" a telephone subscriber who is expected to "answer" the incoming call. However current health service models still depend on the telephone subscriber paradigm which requires a patient to make a telephone call, set up an appointment time, and then the healthcare professional will meet the with or call the patient. This model helps schedule a busy clinician, but requires manual handling of telephone numbers or connection addresses and appointment times. Lastly, where a cloud service maintains a list of users and their contact details, the sharing of user directories and establishing mutual operation between networks requires agreement between organizations to trust each other's procedures and technology.

\subsection{Controlling Reliability, Usability and Quality of Video Conferencing}

Australia has been in the forefront of video conferencing for medical purposes, through a number of initiatives. For example, in 1997, Queensland Health had about 200 video conferencing systems connected by ISDN and was testing high definition systems running over networks faster than a home connection on the NBN. Queensland Health now has a about 2000 systems in over 200 hospitals and community facilities, supporting more than 40 clinical specialties [13] and subspecialties, but per user usage is lower than it could be at 8.9 hours per month per system [14].

One particular issue that video conferencing users in the health sector feel is important is the reliability, usability and quality of video systems. For this reason health organizations often choose to base their video conferencing systems on enterprise grade reliable telecommunication networks which can prioritize the transmission of audio and video (quality of service aware networks) and dedicated endpoints linked to multipoint conferencing bridges.

The ongoing proliferation of consumer grade video conferencing products now poses challenges for health care organizations that were early entrants to the field and built video conferencing systems on dedicated hardware and custom designed networks. Healthcare organizations using this type of system were able to control the quality of service provided by their networks to prevent their use potentially impinging on the performance of other health applications on the same network. ICT managers were also able to carefully control the configuration and operation of these systems. In contrast software based video conferencing which may be implemented 
on many types of device, presents challenges for the maintenance and provision of suitable network capacity. While the health sector may have a large sunk investment in dedicated endpoint technology, the sector is just one market segment for video conferencing suppliers and as technology changes the health sector will have no choice but to adapt to the wider influences of the consumer device and applications market.

\subsection{Comparative Adoption of Voice and Video Communications}

Application of Metcalf's Law [15] provides a powerful incentive for organizations to increase the number of users of a video conferencing network, increase its usage and connect with other organizations. Metcalf's Law states that the value of a network can be approximated to increase at a rate proportional to the number of users. Of course the assumption that equal value may be gained by connecting additional users may depend on the type of user, for instance a general practitioner may have different usage patterns to a consultant medical specialist.

Economic analysis of the growth of networks [16] highlights the points in the growth of a network at which there is more value to an organization to cooperate rather than compete with another rival organization, by either establishing an agreement to interconnect with the other network, or structurally amalgamate by agreement or takeover. This type of analysis coupled with historical comparisons provides some insight into possible future directions for video conferencing and telehealth networks.

The closest historical and technical comparison for video conferencing network evolution is the growth of the telephone network. Telehealth networks using video conferencing are very small by comparison with the telephone and internet so it is of value to understand how the telephone network changed over time. Telephone networks also started small, often created by entrepreneurs and competed for customers within a given geographical area. For instance in the US town of Keokuk:

"When the Bell patents expired in 1893, many exchanges were formed to compete with existing companies. In some cities two or three companies competed, so in order to be connected to all his neighbors, a telephone user had to have two or more telephones, directories, and bills” [17].

Individual telephone companies in the US were numerous (about 6,000 companies) and not large at the turn of century, but subsequently the monopoly Bell system of companies emerged though a process of takeovers that lasted 30 years and untilregulated by government to provide a universal service, on the basis of that:

"The telephone, by nature of its technology, would operate most efficiently as a monopoly providing universal service” [18].

In other countries the public sector played a major role in unifying the telephone system. In the United Kingdom, the Telegraph Act of 1892 nationalized the provision of the long-distance telephony under the General Post Office [19]. In Australia the first state to take over a telephone system was Victoria when in 1887 when the government purchased the Victorian Telephone Exchange Company, shortly followed by the new Commonwealth Government at the time when all Australian states agreed to join together in a federation in 1901 [20] and all telephone systems across the country were nationalized.

In contrast, video conferencing technology has only been developing since the middle part of last century. In 1964 Bell Labs demonstrated an experimental Picturephone system [21] that operated over a copper telephone line. The promise of these early efforts to provide a video conferencing product that would be interchangeable with the telephone came to an end when the rise of the internet broke the technology and business connection with the established telecommunications system providers.

Health informatics standards have not yet played an effective role in simplifying this picture for users. While in 2004 the International Standards Organization (ISO) published a telehealth interoperability standard [22] this standard was based on older pre-internet and early versions of H.323 technology, and has become less important with the development of new technologies. Currently standards organizations such as ISO, ITU and the IETF are now collaborating on WebRTC standards development.

Standards are heavily influenced by commercial considerations. Agreement between vendors to work to one standard does not usually take place until there is a commercial benefit to do so, which in turn depends on their analysis of the benefits of joining in a cooperating network. Vendors that stand to benefit from greater standardization can play a unifying role in the industry. For instance Alcatel-Lucent sells core technology to telecommunications companies and advocates greater use of “standardized” technology such as WebRTC, while stating: “Given that today's global PSTN ... ecosystem has made a social contract of universal reach possible and expected ...the next generation IP communications ecosystem must do no less” [23]. 


\section{Conclusions-Competing Clouds}

Instead of video conferencing phones connected via the (enhanced) telecommunications system, islands of video conferencing businesses have appeared on the internet, marketed as cloud services, that are currently competing on the basis of price and features for users. There is at present no single dominant video conferencing player, although Skype and Facetime would have to be contenders. Technical and commercial factors have supported differentiation of product features and mitigated against cooperation so that users may well have a Facetime account, a Skype account, or another video cloud account on the one machine, with a "social" network of people they can call associated with each account which is not unlike the situation in the US town of Keokuk in 1893 referred to previously.

The experience of the FTH trial in choosing a video conferencing technology which was both a proprietary and a cloud based service (albeit hosted by Flinders University) illustrates the impact of vendor competition on the ability of health organizations to seamlessly connect video conferencing calls between each other, and connect to patients who may be using a variety of video conferencing solutions at home.

Comparison of the current state of the video conferencing market place with the evolution of the telephony system reveals that video conferencing still has a long way to go before it can be considered as easy to use as the telephone. The technology is still relatively immature, market ownership is heavily contested and the influence of standards is small. There are signs that this situation may be improving, however, health organizations that are concerned to improve access and quality of care should seek to influence greater standardization and interoperability though cooperation with one another, the private sector, international organizations and by encouraging governments to play a more active role in this sphere.

\section{Acknowledgements}

The FTH trial was an initiative funded by the Australian Government. The authors would also like to thank all participants in the FTH trial, staff at the Repatriation General Hospital in Adelaide and fellow researchers including, Professor Maria Crotty, Professor David Currow, Kate Swetenham, Associate Professor Jennifer Tieman and Dr. Craig Whitehead.

\section{Declaration of Conflicting Interests}

The authors declare that there are no conflicts of interest.

\section{References}

[1] Crotty, M., Killington, M., van den Berg, M., Morris, C., Taylor, A. and Carati, C. (2014) Telerehabilitation for Older People Using Off-the-Shelf Applications: Acceptability and Feasibility. Journal of Telemedicine and Telecare, 20, 370-376. http://dx.doi.org/10.1177/1357633X14552382

[2] Taylor, A., Morris, G., Tieman, J., Currow, D., Kidd, M. and Carati, C. (2015) Building an Architectural Component Model for a Telehealth Service. E-Health Telecommunication Systems and Networks, 4, 35-44. http://dx.doi.org/10.4236/etsn.2015.43004

[3] International Telecommunications Union (2009) ITU-T H.323 v7 (12/2009). http://www.itu.int/ITU-T/recommendations/rec.aspx?rec=H.323

[4] International Telecommunications Union (2014) H.264: Advanced Video Coding for Generic Audiovisual Services. https://www.itu.int/rec/T-REC-H.264

[5] WebRTC (2014) WebRTC Architecture. http://www.webrtc.org/architecture

[6] Bankoski, J., Wilkins, P. and Xu, Y. (2013) Technical Overview of VP8, an Open Source Video Codec for the Web. In 2011 International Conference on Multimedia and Expo (ICME), 1-6.

[7] Kelly, B. (2013) Vidyo-Google Announcement of VP9 SVC for WebRTC: Why It’s Important. In: No Jitter, 2 March. http://www.nojitter.com/post/240160699/vidyogoogle-announcement-of-vp9-svc-for-webrtc-why-its-important

[8] IETF (2002) SIP: Session Initiation Protocol. https://www.ietf.org/rfc/rfc3261.txt

[9] IETF (2004) The E.164 to Uniform Resource Identifiers (URI) Dynamic Delegation Discovery System (DDDS) Application (ENUM). https://tools.ietf.org/html/rfc3761

[10] Taylor, A., Wade, V., Morris, G., Pech, J., Rechter, S., Kidd, M., et al. (2015) Technology Support to A Telehealth in the Home Service: Qualitative Observations. Journal of Telemedicine and Telecare. http://dx.doi.org/10.1177/1357633X15601523 
[11] Taylor, A., Morris, G., Pech, J., Rechter, S., Carati, C. and Kidd, M.R. (2015) Home Telehealth Video Conferencing: Perceptions and Performance. JMIR mHealth and uHealth, 3, e90. http://mhealth.jmir.org/2015/3/e90

[12] ACCRM (2014) ACRRM Telehealth Provider Directory. http://www.ehealth.acrrm.org.au/provider-directory

[13] Queensland Government (2014) Inquiry into Telehealth Services in Queensland. Report 55, p. 37. http://www.parliament.qld.gov.au/Documents/TableOffice/TabledPapers/2014/5414T5969.pdf

[14] Queensland Government (2014) Inquiry into Telehealth Services in Queensland. Report 55, p. 55. http://www.parliament.qld.gov.au/Documents/TableOffice/TabledPapers/2014/5414T5969.pdf

[15] Metcalf, B. (2013) Metcalfe’s Law after 40 Years of Ethernet. Computer, 46, 26-31. http://dx.doi.org/10.1109/MC.2013.374

[16] Economides, N. (1996) The Economics of Networks. International Journal of Industrial Organization, 14, 673-699. http://dx.doi.org/10.1016/0167-7187(96)01015-6

[17] Telecommunications History Group (2014) Twin City Telephone Company. http://www.telcomhistory.org/vm/historiesTwin.shtml

[18] AT\&T (2015) A Brief History: The Bell System. http://www.corp.att.com/history/history3.html

[19] Ward, K. (2006) A Short History of Telecommunications Transmission in the UK. The Journal of the Communications Network, 5, 30-41.

[20] Vintage Phones (2014) History of Telephone Exchanges in Australia. http://www.vintagephones.com.au/ccp0-display/history-of-the-telephone-exchange-in-australia.html

[21] Paleofuture (2014) A Brief History of the Videophone That Almost Was. http://paleofuture.gizmodo.com/a-brief-history-of-the-videophone-that-almost-was-1214969187

[22] International Standards Organisation (2004) ISO/TR 16506-2:2004(en) Health Informatics-Interoperability of Telehealth Systems and Networks. International Organization for Standardisation, Geneva.

[23] Lucent, A. (2013) WebRTC IMS Systems and WebRTC Proprietary Islands: Innovations Enablement or Paradigm Shift? Technical White Paper.

http://www.tmcnet.com/tmc/whitepapers/documents/whitepapers/2013/8641-alcatel-lucent-webrtc-ims-systems-webrtc -proprietary-islands.pdf 